\title{
Prescrição de medicamentos potencialmente inapropriados em instituições de longa permanência para idosos
}

\author{
Prescription of potentially inappropriate medications in homes for the aged
}

Marcos Hortes N. Chagas ${ }^{1,2}$, Ana Carolina Chini3 ${ }^{3}$ Rebeca Mendes de Paula Pessoa²

\section{PREZADO EDITOR,}

Os critérios de Beers publicados pela Sociedade Americana de Geriatria são utilizados para identificar medicamentos de uso potencialmente inapropriados (MPIs) em idosos'. Em 2012, os critérios foram atualizados considerando método baseado em evidências. Esse instrumento pode ser empregado facilmente por especialistas e não especialistas no auxílio do manejo farmacológico de pacientes idosos, contribuindo para prescrição médica mais adequada e evitando a polifarmácia.

Recentemente, Andrade et al. publicaram um estudo nesta revista apontando a elevada frequência de prescrição desses medicamentos em idosos institucionalizados em um hospital psiquiátrico do interior da Bahia². Nesse estudo, um ou mais medicamentos potencialmente inapropriados estavam presentes em $90 \%$ das prescrições ${ }^{2}$.

Da mesma forma, realizamos um estudo na cidade de Ribeirão Preto, estado de São Paulo, com o objetivo de avaliar a cognição e o comportamento de idosos internados em instituições de longa permanência (ainda não publicado) e encontramos alta frequência de prescrição de MPIs. O estudo foi transversal e realizado em quatro instituições (duas filantrópicas e duas privadas). Foram excluídos pacientes com incapacidade clínica ou neuropsiquiátrica grave. Os dados relativos às medicações foram extraídos diretamente das folhas de prescrições. A pesquisa foi aprovada no Comitê de Ética em Pesquisa para seres humanos (CAAE: 22872813.5.0000.5440).

Foram avaliados 51 idosos, dos quais 64,7\% eram mulheres $(n=33)$. A média de idade foi de 78,1 anos (DP: $\pm 9,8)$ e a média do escore total do Miniexame do Estado Mental foi de 18,2 (DP: $\pm 5,4$ ). MPIs estavam presentes em 52,9\% das prescrições, além disso, duas prescrições apresentavam "fórmulas manipuladas" sem a descrição de seus componentes. As MPIs mais prescritas foram antipsicóticos que estavam presentes em $31,4 \%(n=16)$ das prescrições, sendo a quetiapina a mais presente $(n=7)$. Os benzodiazepínicos encontravam-se prescritos para $25,4 \%(n=13)$ dos idosos. Nesta classe, o diazepam foi o mais prescrito $(n=8)$. Foi encontrada polifarmácia ( $\geq 5$ medicamentos) em 39,2\% dos casos $(n=20)$.

Outros estudos também encontram prevalência semelhante de prescrição de MPIs em idosos, e os psicotrópicos estão entre os mais prescritos ${ }^{3}$. Especialmente em idosos, os profissionais de saúde, incluindo psiquiatras e psicogeriatras, devem ficar atentos para o uso indiscriminado de antipsicóticos e benzodiazepínicos. Os antipsicóticos aumentam a mortalidade em idosos, além de efeitos adversos como parkinsonismo, patologias cerebrovasculares, síndrome metabólica, entre outros, de acordo com as características específicas de cada antipsicótico 4 .

1 Universidade Federal de São Carlos (UFSCar), Centro de Ciências Biológicas e da Saúde. 2 Universidade de São Paulo (USP), Departamento de Neurociências e Ciências do Comportamento.

3 Faculdade de Ciências da Saúde de Barretos Dr. Paulo Prata (FACISB).

Endereço para correspondência: Marcos Hortes N. Chagas Universidade Federal de São Carlos Centro de Ciências Biológicas e da Saúde, Departamento de Gerontologia Rodovia Washington Luís, km 235 13565-905 - São Carlos, SP, Brasil Telefone: +55 (16) 3306-6938

E-mail: setroh@hotmail.com 
Os benzodiazepínicos apresentam potencial elevado de causar tolerância e dependência química e estão associados com maior risco de queda e piora da cognição ${ }^{5}$.

Apesar do alerta, os aspectos singulares de cada paciente devem ser ponderados na prescrição. O estudo de Andrade et al.2 foi realizado em instituição psiquiátrica e a maioria dos participantes apresentava diagnóstico que implicava o uso crônico de medicações. Além disso, os pacientes apresentavam prevalência elevada de outras comorbidades não psiquiátricas, o que também pode ter contribuído para a polifarmácia e a alta frequência de prescrição de MPIs. Da mesma forma, nosso estudo foi realizado em instituições de longa permanência com elevado número de idosos com sintomas neuropsiquiátricos e outras comorbidades clínicas. Desse modo, deve-se destacar que os dados não devem ser extrapolados para a população idosa de forma geral.

Por fim, deve-se ressaltar que os psicotrópicos presentes na lista de MPIs só devem ser usados com indicação precisa e na menor dose possível. Deve-se considerar ainda que medidas não farmacológicas podem ser implementadas para reduzir sintomas comportamentais e psicológicos em idosos.

\section{REFERÊNCIAS}

1. American Geriatrics Society 2012 Beers Criteria Update Expert Panel. American Geriatrics Society updated Beers Criteria for potentially inappropriate medication use in older adults. J Am Geriatr Soc. 2012;60(4):616-31.

2. Andrade KVF de, Silva Filho C da, Junqueira LL. Prescrição de medicamentos potencialmente inapropriados para idosos: um estudo transversal em instituição psiquiátrica. J Bras Psiquiatr. 2016;65(2):149-54.

3. Hill-Taylor B, Sketris I, Hayden J, Byrne S, O'Sullivan D, Christie R. Application of the STOPP/START criteria: a systematic review of the prevalence of potentially inappropriate prescribing in older adults, and evidence of clinical, humanistic and economic impact. J Clin Pharm Ther. 2013;38(5):360-72.

4. Gareri P, De Fazio P, Manfredi VGL, De Sarro G. Use and safety of antipsychotics in behavioral disorders in elderly people with dementia. J Clin Psychopharmacol. 2014;34(1):109-23.

5. Lader M. Benzodiazepines revisited-will we ever learn? Addiction. 2011;106(12):2086-109. 